\title{
Dendrobium Officinale Polysaccharide Attenuates Insulin Resistance and Abnormal Lipid Metabolism in Obese Mice
}

\author{
Jian $\mathrm{Qu}^{1 \dagger}$, Shengyu $\mathrm{Tan}^{2 \dagger}$, Xinyan Xie ${ }^{3}$, Wenqiang $\mathrm{Wu}^{3}$, Haihong $\mathrm{Zhu^{1 }}$, Hang $\mathrm{Li}^{1}$, \\ Xiaobo Liao ${ }^{4}$, Jiaojiao Wang ${ }^{1}$, Zhi-Ang Zhou ${ }^{4}$, Song Huang ${ }^{3 *}$ and Qiong Lu ${ }^{1 *}$
}

${ }^{1}$ Department of Pharmacy, The Second Xiangya Hospital, Central South University, Changsha, China, ${ }^{2}$ Department of Geriatrics, The Second Xiangya Hospital of Central South University, Changsha, China, ${ }^{3}$ Mathematical Engineering Academy of Chinese Medicine, Guangzhou University of Chinese Medicine, Guangzhou, China, ${ }^{4}$ Department of Cardiovascular Surgery, The Second Xiangya Hospital, Central South University, Changsha, China

OPEN ACCESS

Edited by:

Sayeed Ahmad,

Jamia Hamdard University, India

Reviewed by:

Ye Huang,

Xiyuan Hospital CACMS, China Alexandre Abilio De Souza Teixeira,

University of São Paulo, Brazil

${ }^{*}$ Correspondence:

Song Huang

huangnn421@163.com

Qiong Lu

christy_luq@csu.edu.cn

${ }^{+}$These authors have contributed equally to this work

Specialty section: This article was submitted to

Ethnopharmacology,

a section of the journal

Frontiers in Pharmacology

Received: 28 January 2021 Accepted: 01 June 2021

Published: 14 June 2021

Citation:

Qu J, Tan S, Xie X, Wu W, Zhu H, Li H, Liao X, Wang J, Zhou Z-A, Huang $S$ and Lu Q (2021) Dendrobium Officinale

Polysaccharide Attenuates Insulin Resistance and Abnormal Lipid Metabolism in Obese Mice. Front. Pharmacol. 12:659626. doi: 10.3389/fphar.2021.659626
Objectives: Dendrobium officinale polysaccharide (DOP) is the main active ingredient in a valuable traditional Chinese medicine, which exerts several pharmacological activities including hepatoprotection and hypoglycemic effects. However, the effects of DOP on obesity-associated insulin resistance $(\mathbb{R})$ and lipid metabolism remain unknown. This study aimed to investigate the role of DOP in IR and abnormal lipid metabolism in obese mice.

Methods: IR models were established using 3T3-L1 adipocytes, C2C12 myocytes, and primary cultured hepatocytes exposed to palmitate acid. After treatment with DOP, insulinstimulated glucose uptake, glucose release, and AKT phosphorylation was detected. Fasting blood glucose, fasting serum insulin, the glucose tolerance test (GTT), and the insulin tolerance test (ITT) were measured to evaluate IR of obese mice. Lipid analysis was conducted to evaluate the effects of DOP on lipid metabolism in obese mice.

Results: In vitro, DOP treatment ameliorated palmitic acid-induced IR in adipocytes, myocytes, and hepatocytes. DOP regulated cellular insulin sensitivity via the peroxisome proliferator-activated receptor- $\gamma$ (PPAR- $\gamma$ ). Furthermore, administration of DOP significantly reduced the IR and visceral adipose tissue (VAT) inflammation of diet-induced obese (DIO) and the genetically-induced obesity mice (ob/ob) mouse models. In addition, DOP treatment attenuated the high-fat diet (HFD)-induced liver lipid accumulation by reducing liver triglycerides (TG), plasma free fatty acid (FFA), serum cholesterol (TC), and low-density lipoprotein cholesterol (LDL-C) levels, while increasing HDL-C levels.

Conclusion: DOP could improve obesity-associated IR and abnormal lipid metabolism through its activities on PPAR- $\gamma$, and may serve as a potential therapeutic agent for obesity-associated insulin resistance and lipid metabolism disorder.

Keywords: abnormal lipid metabolism, Dendrobium officinale polysaccharide, insulin resistance, obesity, peroxisome proliferator-activated receptor- $\gamma$

Abbreviations: DIO, Diet-induced obese; DOP, Dendrobium officinale polysaccharide; FFA, Free fatty acid; GTT, Glucose tolerance test; HDL-C, High-density lipoprotein cholesterol; HFD, High-fat diet; HOMA-IR, Homeostasis model assessment of insulin resistance; IR, Insulin resistance; ITT, Insulin tolerance test; LDL-C, Low-density lipoprotein cholesterol; PPAR- $\gamma$, Peroxisome proliferator-activated receptor- $\gamma$; TC, Serum cholesterol; TG, Triglycerides; VAT, Visceral adipose tissue. 


\section{INTRODUCTION}

With rapid economic growth, obesity is becoming an increasing health concern worldwide. Understandably, obesity was defined as a disease by the Obesity Society (TOS) in 2008 (Belkina and Denis, 2010; Jastreboff et al., 2019). Obesity is characterized by increased circulating fatty acid levels resulting from excessive lipolysis and absorption of basal adipose tissue, which in turn promote the development of ectopic lipid deposition and insulin resistance (IR) (Crowe et al., 2009). Consistently, studies have shown that obesity is a risk factor for metabolic syndrome, which includes IR and dyslipidemia, impaired fasting blood glucose, and hyperinsulinemia (Laaksonen et al., 2002; Meigs et al., 2006). In addition, obesity increases the risk and mortality of many diseases Council of the Obesity (2008), González-Domínguez et al. (2020), especially type 2 diabetes, cardiovascular disease, hypertension, and dyslipidemia (De Lorenzo et al., 2019).

IR refers to the reduction of insulin sensitivity and response to insulin target organs and tissues (liver, adipose tissue, skeletal muscle) due to varied reasons (Samuel and Shulman, 2012). This leads to a decrease in glucose uptake and utilization. Therefore, the body will compensatively secrete excess insulin to maintain the stability of blood sugar, leading to hyperinsulinemia, which in turn leads to dyslipidemia. Dyslipidemia manifests as increased total cholesterol (TC), triglycerides (TG), and free fatty acids (FFA), normal or slightly increased low-density lipoprotein cholesterol (LDL-C), and decreased high-density lipoprotein cholesterol (HDL-C) (Klop et al., 2013). Moreover, the symptoms of IR are impaired glucose absorption in muscles and increased gluconeogenesis in the liver during fasting and postprandial conditions, leading to hyperglycemia (Moller and Kaufman, 2005). Insulin-sensitive tissues (adipose tissue, heart, and liver) are significantly affected by obesity at biomolecular and functional levels (Barazzoni et al., 2018). Excessive fat accumulation can change the function of adipose organs and leads to obesity-related diseases (Ling and Ronn, 2019). In addition, a close relationship between the mechanisms implicated in lipid metabolic disorders and IR has also been reported (Savage et al., 2007). Further, obesity and IR are closely related to inflammation of metabolic tissues, including the liver, muscle, and adipose tissue (Winer et al., 2016; Wu and Ballantyne, 2017; Ghorpade et al., 2018). Excessive fat accumulation and abnormal lipid metabolism caused by obesity induce the transformation of macrophages from the anti-inflammatory M2 polarized state to the pro-inflammatory M1 polarized state, leading to VAT inflammation, thus promoting the development of obesity-related IR (Xu et al., 2003; Lumeng et al., 2007; Kojta et al., 2020). At the same time, IR further aggravates lipid metabolism disorders, leading to a series of metabolic disorders such as diabetes and cardiovascular diseases. The specific manifestations of visceral adipose tissue (VAT) inflammation are increased levels of proinflammatory cytokines such as tumor necrosis factor (TNF)- $\alpha$ and interleukin (IL)-6 Hotamisligil et al. (1993), Kern et al. (2001), and decreased levels of anti-inflammatory cytokines IL-10 and IL-4 in obesity-related IR patients (Esposito et al., 2003). Therefore, the prevention or treatment of IR and lipid metabolism disorders may be an effective way to inhibit obesityrelated diseases.

Dendrobium officinale Kimura et Migo is a valuable traditional Chinese medicine that has been used to treat diabetes, obesity, rheumatoid arthritis, and many other diseases. It has been reported that Dendrobium officinale Kimura et Migo can inhibit the effects of oxidative stress and pro-inflammatory cytokines (Teixeira da Silva et al., 2015). Oxidative stress and inflammation may also lead to obesity-related IR (Xu et al., 2003; Gortan Cappellari et al., 2016). Dendrobium officinale polysaccharide (DOP) is the main active component of Dendrobium officinale Kimura et Migo, which exerts pharmacological activities including antioxidation, lowering blood lipids and blood sugar levels, and hepatoprotection (Huang et al., 2016). In type 2 diabetic rats, DOP treatment reduced hepatic lipid metabolism disorders and alleviated symptoms of hepatic lipid accumulation (Yang et al., 2020). These studies indicated that DOP is expected to be a novel therapeutic agent for obesity-related diseases including IR and abnormal lipid metabolism. However, to date, there are almost no available mechanistic studies investigating mechanistic studies on the effects of DOP in IR and abnormal lipid metabolism. Meanwhile, the mechanism of DOP in obesity-related IR and abnormal lipid metabolism is still unclear.

The purpose of this study was to explore the effects and mechanisms of action of DOP on obesity-related IR and lipid metabolism disorders. First, at the cellular level, we measured glucose uptake, glucose output, and Akt phosphorylation in insulin-stimulated hepatocytes, muscle cells, and adipocytes, respectively. The results showed that DOP treatment could improve IR-induced obesity. Subsequently, we conducted a mechanism study and found that DOP mainly acted on peroxisome proliferator-activated receptor- $\gamma$ (PPAR- $\gamma$ ) signaling to regulate insulin sensitivity in diet-induced obesity (DIO) mice. We evaluated how DOP could improve IR in DIO mice by assessing fasting blood glucose and fasting serum insulin levels, the glucose tolerance test (GTT), the insulin tolerance test (ITT), and the inflammation related indicators of VAT. Furthermore, lipid analysis showed that DOP attenuated the abnormal lipid metabolism in DIO mice. We also validated the therapeutic effects of DOP in the genetically-induced obesity mice (ob/ob mice) model. Overall, we expect that our study can provide a basis for the clinical application of DOP in obesity-related IR and lipid metabolism disorders.

\section{MATERIALS AND METHODS}

\section{Animal Models}

To establish a diet-induced obese (DIO) mice model, two-monthold male C57BL6 mice were fed a high-fat diet (HFD, 60\% fat, $20 \%$ protein, and $20 \%$ carbohydrate) for 3 months. Ob/ob mice are a classical experimental model of obesity-induced IR as evidenced by a plethora of studies. Ob/ob mice harbor a recessive mutation in leptin and are characterized by hyperphagia, obesity, hyperinsulinemia, insulin-resistance (IR), and hyperglycemia; thus, they are commonly used as a model for 

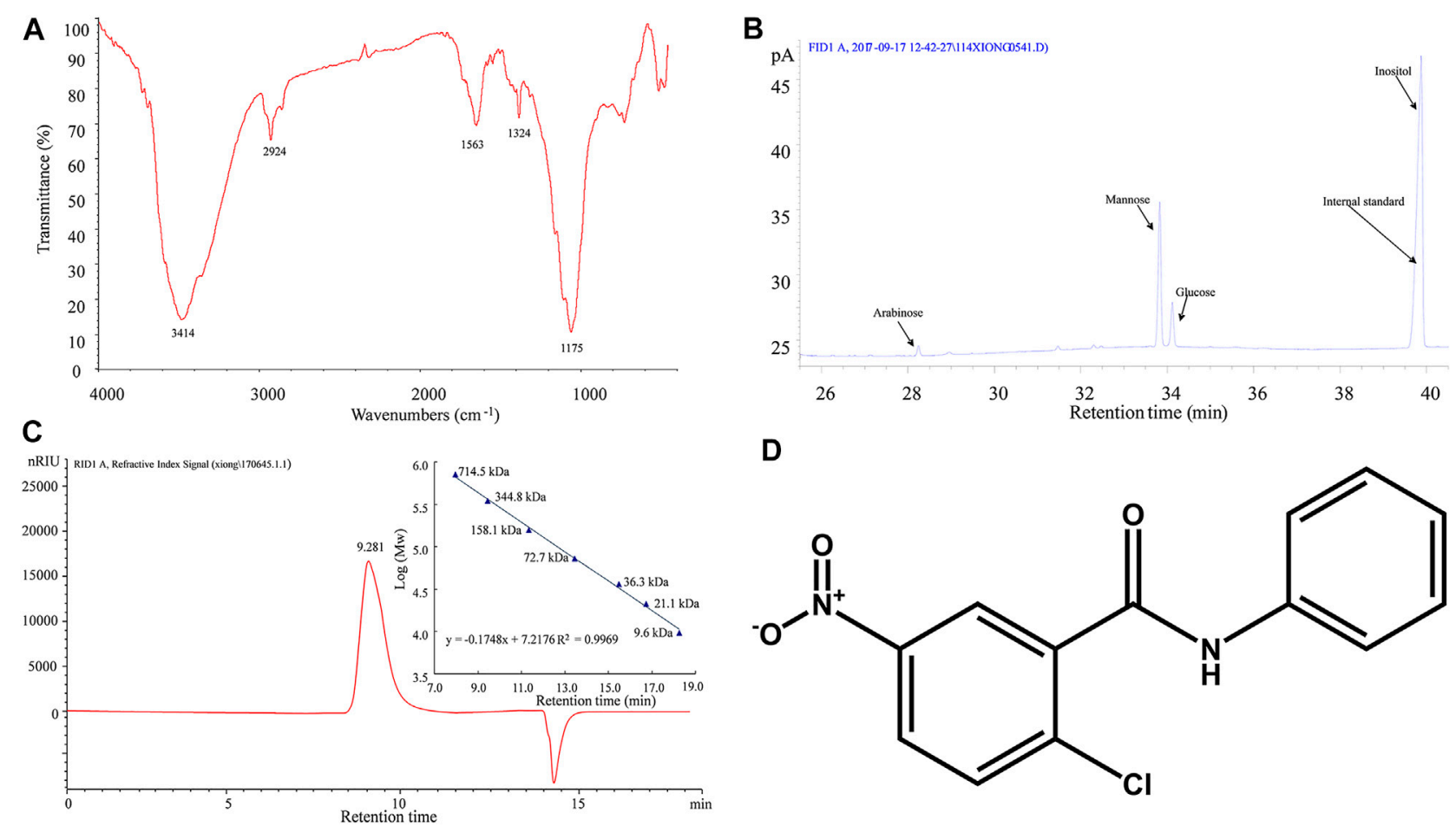

FIGURE 1 | Characterization of DOP (A) The FT-IR spectrogram of DOP (B) GC chromatogram of monosaccharide composition showed that DOP is allocated to $\mathrm{O}-\mathrm{H}$ stretching vibrations, $\mathrm{C}-\mathrm{H}$ stretching vibrations and $\mathrm{C}-\mathrm{C}$ or $\mathrm{C}-\mathrm{O}$ stretching vibrations, respectively. These is the characteristic absorption profile of polysaccharides. The monosaccharide composition of DOP was mannose, glucose, and arabinose with a molar ratio of 5.55:1:0.12. (C) The average molecular weight of DOP was $393.8 \mathrm{kDa}$ by HPLC profiles of molecular weight measurement. (D)The chemical structure diagram of the PPAR $\gamma$ antagonist GW9662.

studies of diabetes and obesity. Ob/ob mice were purchased from Hunan SJA Laboratory Animal Co., Ltd. (license number: SCXK 2019-0004). All animals were housed under 12-h light/dark cycles and were provided unrestricted access to food and water unless otherwise specified.

For in vivo DOP treatment, after 3 months of a HFD, the DIO mice were divided into two groups, the control group and the experimental group, the control group received normal saline (NS) orally, and the experimental group was administrated DOP $(150 \mathrm{mg} / \mathrm{kg}$ ) orally, once daily for 3 months. The DOP involved in this study was prepared by the laboratory of Mathematical Engineering Academy of Chinese Medicine, Guangzhou University of Chinese Medicine (Liang et al., 2018; Liang et al., 2019). The proportion of total polysaccharides in the DOP was $93.80 \%$. Moreover, the FT-IR spectrogram showed that DOP has an intense and broad absorption peak around $3414 \mathrm{~cm}-1$, a weak absorption peak at $2924 \mathrm{~cm}-1$ and $1324 \mathrm{~cm}-1$, and an asymmetrical extension at $1175 \mathrm{~cm}-1$ (Figure 1A). And GC chromatogram of monosaccharide composition showed that DOP had the characteristic absorption profile of polysaccharides. DOP is composed of mannose, glucose, and arabinose with a molar ratio of 5.55:1:0.12 (Figure 1B). Meanwhile, the average molecular weight of DOP determined by HPLC profiles of molecular weight measurement was $393.8 \mathrm{kDa}$ (Figure 1C). The structure composition of DOP showed that its formulation was identical to the DOP prepared by Hua (Hua et al., 2004).

All protocols pertaining to animal care and experiments were reviewed and approved by the Institutional Animal Care and Use Committee of the Laboratory Animal Research Center at Xiangya Medical School of Central South University, China.

\section{Cell Culture and Treatment}

Primary hepatocytes, C2C12 myoblasts, and 3T3-L1 preadipocytes were cultured in a humidified incubator at $37^{\circ} \mathrm{C}$ and supplemented with 5\% CO2. Standard protocols were utilized to induce differentiation of these cells. To establish IR models, primary hepatocytes, $\mathrm{C} 2 \mathrm{C} 12$, myoblasts and 3T3-L1 preadipocytes were treated with $0.5 \mathrm{mmol} / \mathrm{L}$ palmitate acid (PA; Sigma) for $24 \mathrm{~h}$. Glucose levels in the medium were measured to determine whether the IR model was successful. For in vitro DOP treatment, $200 \mu \mathrm{g} / \mathrm{ml}$ DOP was added to the culture medium for $48 \mathrm{~h}$.

\section{Cell Viability}

The Cell Counting Kit-8 (CCK-8) was used to assess the viability of 3T3-L1 adipocytes after DOP treatment, as per the manufacturer's protocol. Absorbance was measured at $450 \mathrm{~nm}$ via a microplate reader (Thermo Electron Corp). 
TABLE 1 | Primer sequences used for real-time PCR analysis.

\begin{tabular}{|c|c|c|}
\hline Gene & Forward primer & Reverse primer \\
\hline Actin (mouse) & GGCTGTATTCCCCTCCATCG & CCAGTTGGTAACAATGCCATGT \\
\hline PPAR- $\gamma$ (mouse) & GGAAAGACAACGGACAAATCAC & TACGGATCGAAACTGGCAC \\
\hline TNFa (mouse) & TATGGCTCAGGGTCCAACTC & CTCCCTITGCAGAACTCAGG \\
\hline IL-6 & AGTTGCCTTCTTGGGACTGA & CAGAATTGCCATTGCACAAC \\
\hline IL-10 & GCCCTTCCTATGTGTGGTTTG & TTGAGTTCCGTACTGTाGAGG \\
\hline IL-4 & CCCCAGCTAGTTGTCATCCTG & CAAGTGATIITGTCGCATCCG \\
\hline
\end{tabular}

Glucose Uptake and Glucose Output Assay The glucose uptake and glucose output assays were performed as described previously (Su et al., 2019). The medium glucose levels were measured by a Glucose Assay Kit (Abcam, ab65333) according to the manufacturer's instructions.

\section{Western Blot and qRT-PCR Analysis}

Western blotting analysis was conducted as previously described (Li et al., 2009). Primary antibodies p-AKT (CST9272s; Dilution 1:1,000) and AKT (CST9272s; Dilution 1:1,000) were purchased from Cell Signaling Technology, and PPAR- $\gamma$ (ab209350, Dilution 1:1,000) was purchased from Abcam. All primary antibodies were incubated at $4^{\circ} \mathrm{C}$ overnight, the secondary antibodies were diluted to 1:5,000, and specific proteins were visualized by ECL Plus. For qRT-PCR analysis, the total RNA from cultured cells were isolated by TRIzol (Thermo Fisher Scientific). The list of primers used for real-time PCR analysis are described in Table 1.

\section{Blood Glucose, Serum Insulin, Insulin Tolerance Test, Glucose Tolerance Test, and HOMA-IR Index}

The measurement of blood glucose levels and serum insulin, and the performance of the ITT and GTT, have been reported previously (Ying et al., 2017; Xiao et al., 2020). The following formula was used to calculate the homeostasis model assessment of IR (HOMA-IR) index: [fasting blood glucose levels ( $\mathrm{mmol} / \mathrm{L})$ ] $\times$ [fasting serum insulin levels $(\mu \mathrm{U} / \mathrm{ml})] / 22.5$ (Su et al., 2019).

\section{Lipid Analysis}

Approximately, $0.1 \mathrm{~g}$ liver was homogenized in phosphatebuffered saline (PBS) solution (1:9 ratio). The mixture was centrifuged at $2,500 \mathrm{rpm}$ for $10 \mathrm{~min}$ at $4^{\circ} \mathrm{C}$ after which the supernatant was collected. The cytoplasm of L02 cells was collected with $2 \%$ Triton X-100. Hepatic and hepatocellular TG were quantified using chemical reagent kits from Nanjing Jiancheng Bioengineering Institute (Nanjing, China). In addition, serum lipids including TC, TG, LDL-C, and HDL-C levels were determined using kits from Nanjing Jiancheng Bioengineering Institute (Nanjing, China). Plasma-free fatty acid (FFA) levels were measured enzymatically using a kit from WAKO Chemicals. Absorbance values of all samples were measured using a spectrophotometric system.

\section{Hematoxylin and Eosin Staining and Oil Red O Staining}

Livers samples (4-5 micron in thickness) were placed in cassettes and submerged in $10 \%$ formalin solution overnight. Samples were processed in a dehydrating ethanol gradient, followed by xylene incubation and paraffin embedding. Serial $8-\mu \mathrm{m}$ sections were used for hematoxylin and eosin (H\&E) staining and Oil Red O staining.

\section{Quantification and Statistical Analysis}

All data are presented as mean \pm SD. For comparisons between two groups, a two-tailed Student's t-test was used. Comparisons between multiple groups were performed using ANOVA followed by Bonferroni's post-hoc correction. A $p$-value $<0.05$ was considered statistically significant.

\section{RESULTS}

\section{DOP Ameliorated Cellular Insulin Resistance In Vitro}

The cytotoxicity of DOP was assessed by the CCK- 8 assay, which revealed DOP did not affect cell viability at concentrations of 100 , 200, and $400 \mu \mathrm{g} / \mathrm{ml}$ (Figure 2A). To explore whether DOP participated in the regulation of insulin sensitivity, we treated PA-induced adipocytes, myocytes, and hepatocytes with DOP. As expected, DOP treatment significantly promoted the insulinstimulated glucose uptake of 3T3-L1 adipocytes and $\mathrm{C} 2 \mathrm{C} 12$ myocytes (Figures 2B,C), while the glucose output of primary cultured hepatocytes decreased (Figure 2D). In addition, the insulin-stimulated AKT phosphorylation of 3T3-L1 adipocytes, $\mathrm{C} 2 \mathrm{C} 12$ myocytes, and primary cultured hepatocytes was increased following DOP treatment (Figure 2E). Taken together, these results suggested that DOP ameliorated cellular IR of adipocytes, myocytes, and hepatocytes in vitro.

\section{DOP Regulated Cellular Insulin Sensitivity by Activating PPAR- $\gamma$}

Pharmacological activation of PPAR- $\gamma$ has emerged as an effective method for treating diabetes. We found that the transcript levels of PPAR- $\gamma$ mRNA in 3T3-L1 adipocytes, $\mathrm{C} 2 \mathrm{C} 12$ myocytes, and primary cultured hepatocytes treated with DOP were elevated (Figures 3A-C). Western blotting analysis further confirmed that DOP could promote the protein expression levels of PPAR- $\gamma$ (Figure 3D). These 


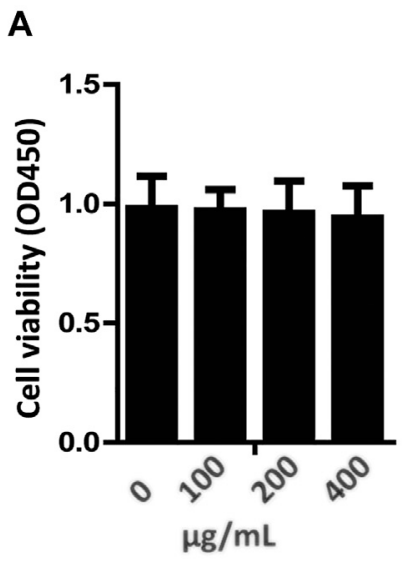

D

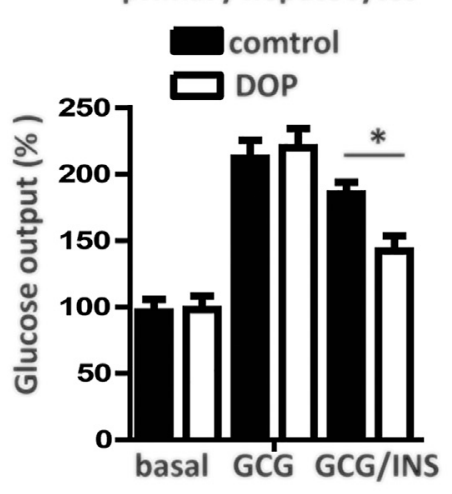

B

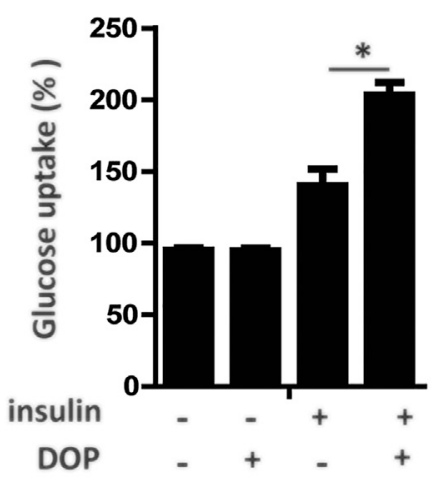

C

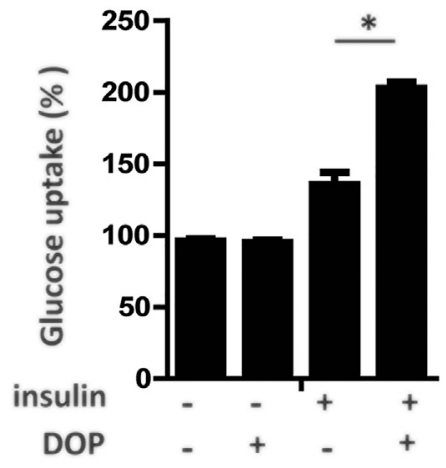

E

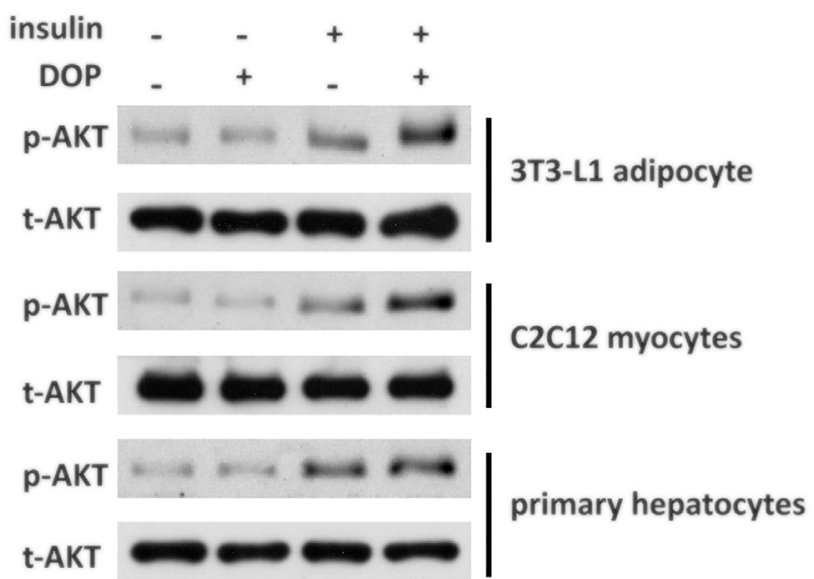

FIGURE 2 | DOP ameliorated cellular insulin resistance in vitro. (A) 3T3-L1 adipocytes were seeded into 96-well plates at a density of $8 \times 103$ cells/well and were treated with different concentrations of DOP for $48 \mathrm{~h}$. Cell viability was determined using CCK-8 assay. (B-D) Influence of DOP on glucose uptake of 3T3-L1 adipocytes and C2C12 myocytes as well as glucose output of primary cultured hepatocytes (E) Insulin stimulated AKT phosphorylation in 3T3-L1 adipocytes, C2C12 myocytes, and primary cultured hepatocytes. Data are presented as mean \pm SD. Statistical significance was determined using analysis of variance (ANOVA) for A, and Student's t-test for B, C, and D.* means $p<0.05$.

observations suggested that DOP increased the expression of PPAR- $\gamma$.

To validate whether DOP regulated cellular insulin sensitivity through the activation of PPAR- $\gamma$, we treated PA-induced adipocytes and myocytes with GW9662, a PPAR- $\gamma$ antagonist. GW9662 is a potent and selective PPAR- $\gamma$ antagonist with an IC50 of $3.3 \mathrm{nM}$, showing 10 and 1000-fold selectivity over PPARa and PPAR- $\delta$, respectively. The chemical structure is shown in Figure 1D. The effects of DOP on 3T3-L1 adipocytes and C2C12 myocytes was abrogated by GW9662, which indicated that DOP failed to improve the insulin sensitivity if PPAR- $\gamma$ was inhibited (Figures 3E,F). Taken together, these results indicated that DOP regulated cellular insulin sensitivity by activating PPAR- $\gamma$.

\section{Administration of DOP Reduced the Insulin Resistance of Obese Mice}

To explore the therapeutic potential of DOP on the IR of obese mice, we treated obese mice with normal saline (NS) or DOP.
Notably, mice treated with DOP had lower fasting blood glucose levels and fasting serum insulin (Figures 4A,B). The HOMA-IR index was also lowered in the DOP-treated group (Figure 4C). Moreover, DOP could also suppress VAT inflammation. The levels of pro-inflammatory cytokines IL- 6 and TNFa were also decreased, while the anti-inflammatory cytokines IL-10 and IL-4 were increased (Figure 4D). Furthermore, the results of the GTT and ITT revealed that glucose tolerance and clearance in mice were increased after administration of DOP (Figures 4E,F). Together, these findings suggested that administration of DOP reduced IR and adipose tissue inflammation in obese mice.

\section{DOP Impaired Lipid Metabolism Disorder in Obese Mice}

To further explore the effect of DOP on lipid metabolism in obese mice, serum, and hepatic lipid levels were assayed. DOP-treated mice exhibited significantly lower TG levels in the liver when compared with untreated mice (Figure 5A). In addition, FFA, 
A

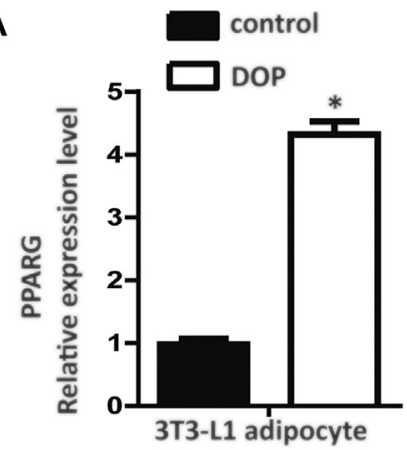

D

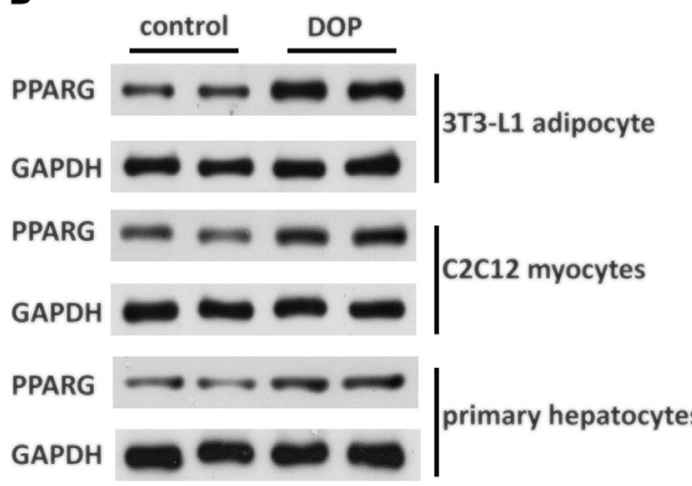

B

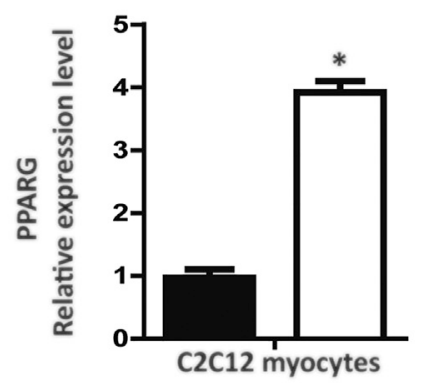

E

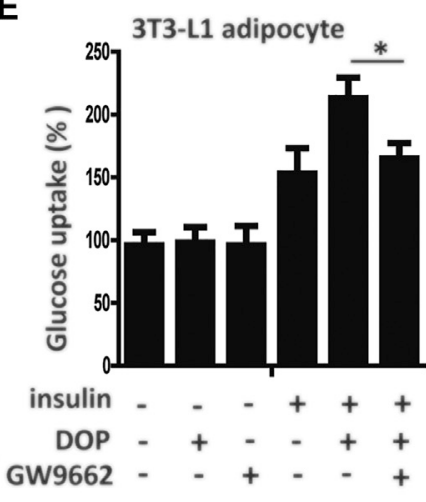

C

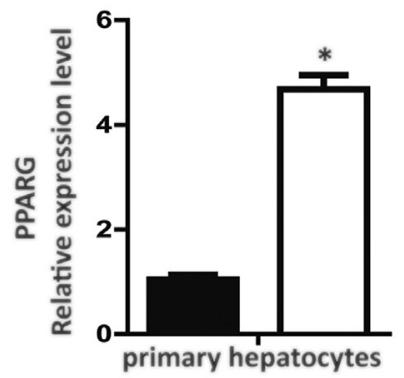

$\mathbf{F}$

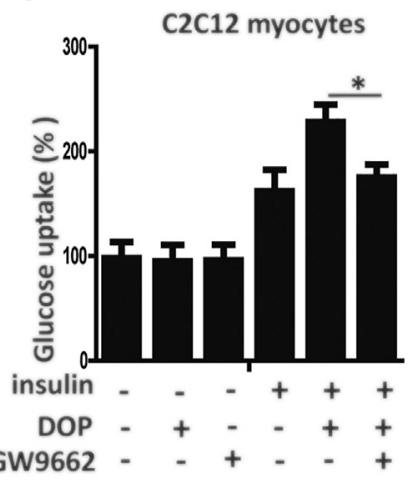

FIGURE 3|DOP regulated cellular insulin sensitivity by activating PPAR- $\gamma$ (A-C) Expression levels of PPAR- $\gamma$ in 3T3-L1 adipocytes, C2C12 myocytes, and primary cultured hepatocytes treated with DOP (200 $\mathrm{\mu g} / \mathrm{ml}, 48 \mathrm{~h})$ (D) Western blot analysis of the protein levels of PPAR- $\gamma$ of 3T3-L1 adipocytes, C2C12 myocytes, and primary cultured hepatocytes treated with DOP. (E-F) Effects of GW9662 (10 $\mu \mathrm{M}, 24 \mathrm{~h})$ on insulin-stimulated glucose uptake of 3T3-L1 adipocytes and C2C12 myocytes. Data are presented as mean \pm SD. Statistical significance was calculated by two-tailed Student's t-test. ${ }^{*}$ means $p<0.05$.

serum TC, TG, and LDL-C levels in DOP-treated mice were also lower than in the control group (Figures 5B-E). Importantly, DOP treatment was effective in increasing HDL-C levels (Figure 5F). The H\&E staining and Oil Red O staining of the liver samples indicated that compared with the control group, the lipid levels and volumes were decreased in DOP treatment group in ob/ob mice (Figure 5G). Taken together, these results showed that DOP could ameliorate lipid metabolism disorders in obese mice.

\section{DOP Treatment Alleviated Insulin Resistance in $\mathrm{Ob} / \mathrm{Ob}$ Mice}

To further confirm the therapeutic effect of DOP, ob/ob mice were orally administered DOP as mentioned above. Similar to the observations in obese mice, ob/ob mice treated with DOP also exhibited decreased fasting blood glucose levels, fasting serum insulins, and a lower HOMA-IR index (Figures 6A-C). In addition, VAT inflammation was also restrained by DOP treatments exhibited by reduced levels of inflammatory cytokines (IL-6 and TNFa) and elevated expression of antiinflammatory cytokines (IL-10 and IL-4) (Figure 6D). The results from the GTT and ITT indicated that the application of DOP could improve the glucose intolerance of ob/ob mice
(Figures 6E,F). Thus, all these results indicated that DOP treatment could alleviate IR and adipose tissue inflammation in ob/ob mice.

\section{DISCUSSION}

Obesity is the basis of IR, which is a potential cause of a complex metabolic syndrome, including hypertension, elevated fasting blood glucose, low HDLC, and elevated TG levels (Beale, 2013). Therefore, there is an urgent need to identify potential therapeutic agents and suitable targets to attenuate IR and abnormal lipid metabolism in obese patients. In this study, we confirmed the potential therapeutic target of PPAR- $\gamma$ in obese mice and the role of DOP in attenuating IR and abnormal lipid metabolism.

IR is defined as the decreased sensitivity to insulin and response of insulin to target organs and tissues (liver, adipose tissue, skeletal muscle) (Samuel and Shulman, 2012). IR can lead to an increase in intracellular glucose concentration and a decrease in glucose uptake. However, for the liver, IR is characterized by an inability to inhibit liver glucose production and glycogen decomposition (Randle et al., 1963; Randle et al., 1964; Randle et al., 1965). AKT protein kinase in adipocytes, 
A

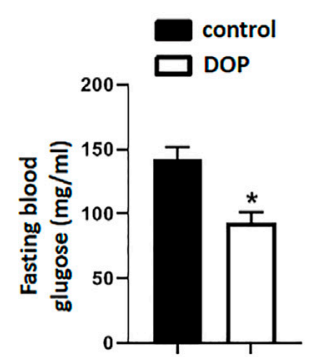

B

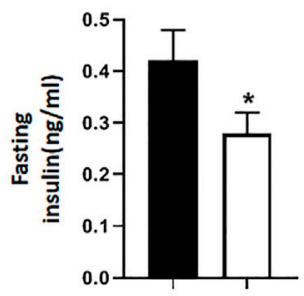

C

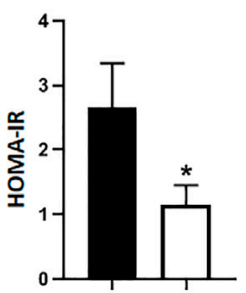

D

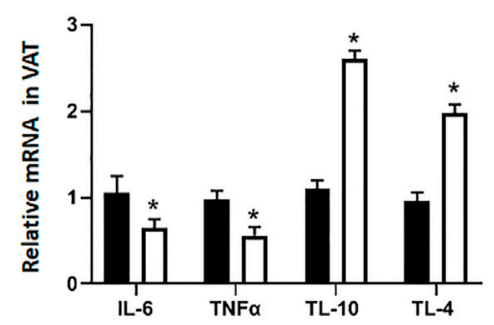

E

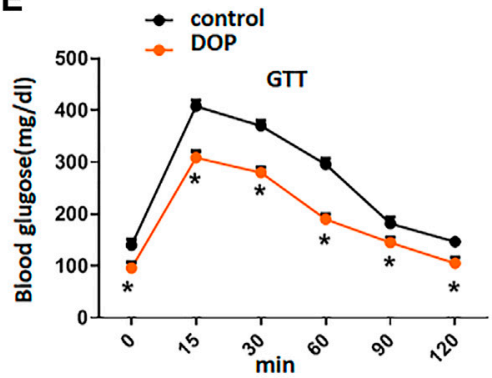

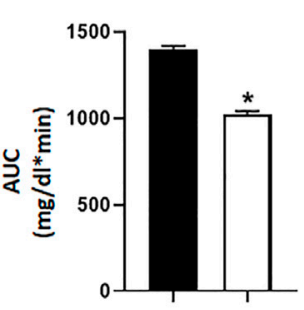

F
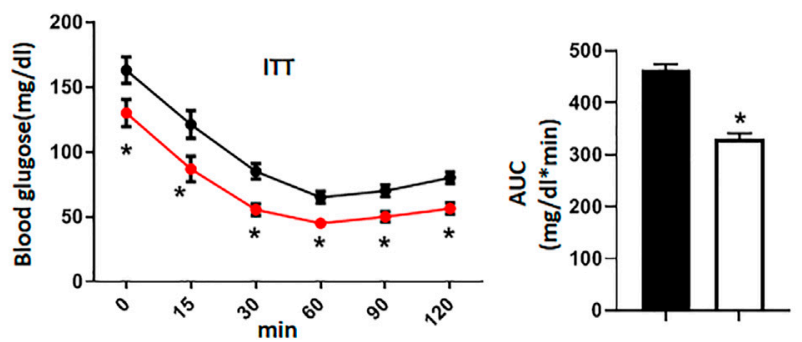

FIGURE 4 | Administration of DOP reduced insulin resistance in obese mice (A-C) The fasting blood glucose levels, fasting serum insulin, and HOMA-IR index of obese mice administered NS or DOP (D) Inflammation-related mRNA levels of VAT in obese mice treated with NS or DOP (E-F). The GTTs and ITTs in obese mice treated with NS or DOP ( $n=5$ per group). AUC: area under curve. Data are presented as mean \pm SD. Statistical significance was calculated using the two-tailed Student's t-test. * means $p<0.05$.

muscle cells, and liver cells is the key kinase regulating glucose homeostasis (Czech, 2017). Previous studies have found that fatty acid stimulation is an early event of IR (Lee et al., 2014). In this study, we used PA to treat adipocytes, myocytes, and hepatocytes to establish IR models of the three cell types. We then measured glucose uptake or output and insulin-stimulated AKT phosphorylation in these insulin-resistant cells with or without DOP treatment. As expected, DOP treatment significantly promoted the insulin-stimulated glucose uptake of 3T3-L1 adipocytes and $\mathrm{C} 2 \mathrm{C} 12$ myocytes, while the glucose output from hepatocytes decreased. Therefore, it is likely that DOP is an agent for potential treatment or prevention of IR.

Multiple mechanisms are involved in the IR of type 2 diabetes. PPAR- $\gamma$ is a subfamily of nuclear receptors. Activation of PPAR- $\gamma$ has been reported to improve blood glucose control and systemic insulin sensitivity in patients with type 2 diabetes (Semple et al., 2006). The activation of nuclear receptor PPAR- $\gamma$ in adipose tissue, liver, and muscle is a determinants of insulin sensitivity (Lu et al., 2011; Germoush et al., 2019). In accordance with previous studies, our results showed that DOP could up-regulate the expression of PPAR- $\gamma$ in adipocytes, myocytes, and hepatocytes.

IR can lead to hyperinsulinemia and impaired fasting blood glucose (elevated fasting blood glucose). Further, ITT, and GTT are also effective methods to evaluate IR (Guerre-Millo et al., 2001). IR can be diagnosed clinically by a homeostasis model of IR (HOMA-IR) based on fasting blood glucose and fasting serum insulin levels (Yee et al., 2019). To further explore the therapeutic effects of DOP on IR, we conducted in vivo experiments using and obese IR mouse model. The results showed that the levels of fasting blood glucose and fasting serum insulin in the DOPtreated group were significantly lower than those in the control group. Through the ITT and GTT, we found that the blood glucose concentration of mice treated with DOP decreased significantly, which indicated that DOP treatment could significantly increase the glucose tolerance of obese mice and clearance rate of insulin to blood glucose. Furthermore, an increase in obesity-induced lipid storage leads to adipose tissue dysfunction and promotes adipocyte secretion of proinflammatory cytokines, including TNF- $\alpha$ and IL-6. Moreover, due to the transformation of M2 macrophages to M1 in obesity tissues, the expression of anti-inflammatory cytokines IL-10 and IL-4 secreted by M2 macrophages is decreased (Taylor, 2021). Changes in the expression of these markers lead to VAT inflammation, which in turn promotes IR. However, our study showed that DOP treatment can reverse the changes in proinflammatory and anti-inflammatory cytokines caused by obesity. These results once again confirmed the therapeutic effects of DOP on obesity-induced IR.

Typical obesity-induced dyslipidemia includes elevation in TC, TG, and FFA levels; normal or mildly elevated LDL-C; and decreased HDL-C levels (Klop et al., 2013). Multiple studies have shown that these changes in lipid metabolism are closely related to IR (Reaven et al., 1988; Frayn, 1993; Boden et al., 1994; Ormazabal et al., 2018). Therefore, we analyzed the levels of serum and liver lipids in obese mice with or without DOP treatment. The FFA and serum TC, TG, and LDL-C levels were lower and the serum HDL-C levels were higher in DOPtreated mice than control mice. These results were similar to the study by Yang et al. study, whereby DOP treatment reduced the 


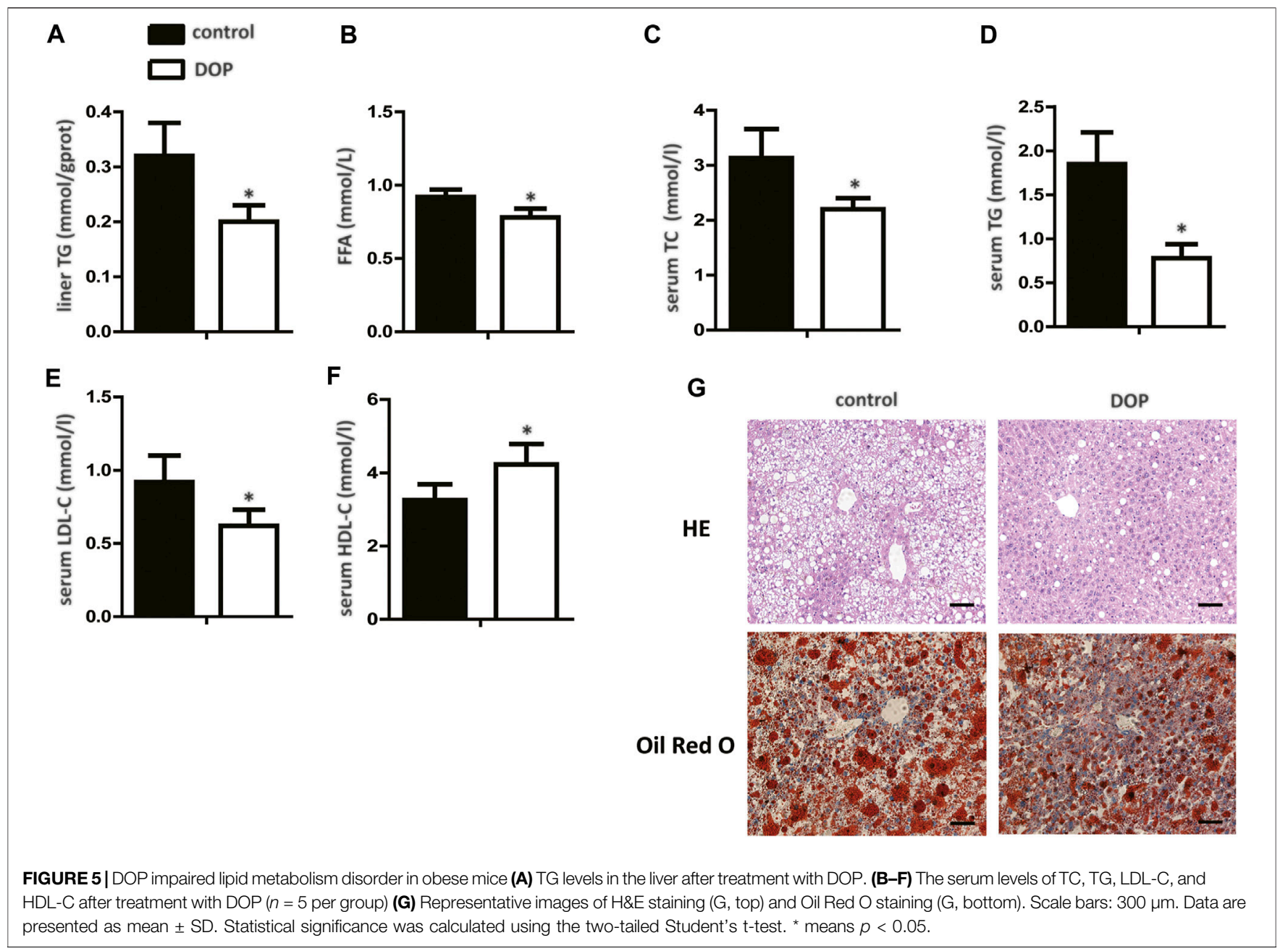

metabolic disorder of liver lipids (fatty acids, TG, and glycerolipids) and reduced the symptoms of lipid accumulation in the liver of type 2 diabetic rats (Yang et al., 2020). Thus, we believe that DOP treatment can improve disorders of obesity-induced lipid metabolism.

Interestingly, only a handful of studies have been published in areas related to DOP and obesity to date. The present study investigated whether DOP could improve IR and lipid metabolism disorders in DIO mice. In order to better demonstrate the therapeutic effects of DOP, we used the ob/ob mouse, a genetic model of obesity. Leptin-deficient (ob/ob) mice are an excellent model of obesity and IR Tomita et al. (1992) and are characterized by elevated insulin and glucose levels and by elevated plasma TG and TC, dyslipidemia, and IR (Dubuc, 1976; Fellmann et al., 2013). Concordant with DIO mice, DOP treatment significantly improved glucose tolerance and insulin clearance of blood glucose in ob/ob mice. Remarkably, DOP has also been shown to inhibit VAT inflammation and liver lipid deposition in the ob/ob mouse model of hereditary obesity. Further, this model was used to further confirm the therapeutic effects of DOP on obesity-related IR and lipid metabolism disorders.
There is a limitation to be noted in our study. Hyperinsulinemic-euglycemic clamp studies are the "gold standard" for evaluating IR, but in our study, we did not adopt this method due to the limitations of the experimental conditions. Nonetheless, an important strength of our study is that it is the first to confirm that DOP can reduce IR and improve abnormal lipid metabolism in obese mice.

In conclusion, DOP can improve insulin sensitivity by upregulating the expression of PPAR- $\gamma$, thus improving obesityrelated IR. In addition, DOP can treat disorders of lipid metabolism in obese mice. To our knowledge, our study is the first to confirm that DOP can reduce IR and improve abnormal lipid metabolism in obese mice, which provides a novel therapeutic option for the treatment of obesity-related IR and lipid metabolism disorders.

\section{DATA AVAILABILITY STATEMENT}

The original contributions presented in the study are included in the article/Supplementary Material, further inquiries can be directed to the corresponding authors. 

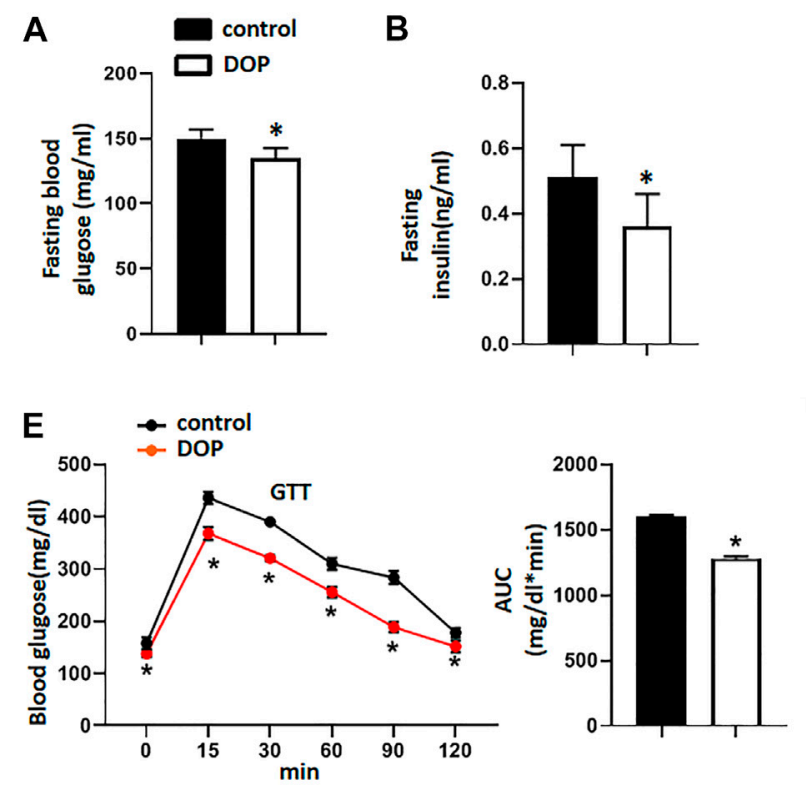
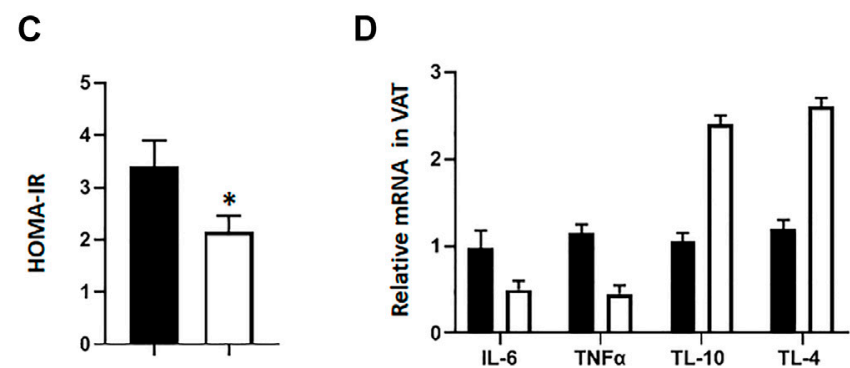

$\mathbf{F}$
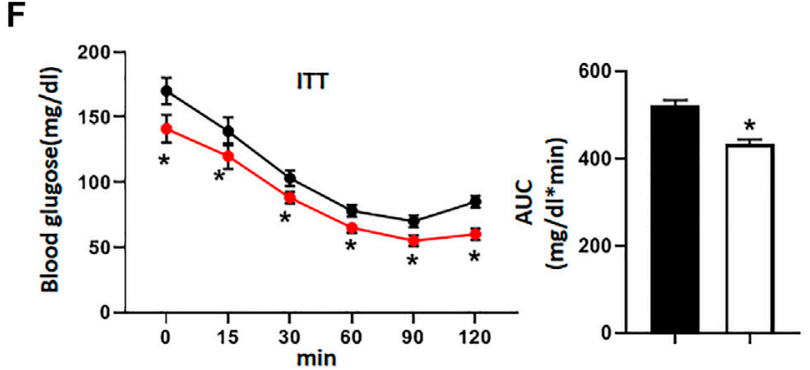

FIGURE 6 | DOP treatment alleviated insulin resistance in ob/obmice (A-C) The fasting blood glucose levels, fasting serum insulin, and HOMA-IR index of ob/ob mice administered NS or DOP (D) Inflammation-related mRNA levels of VAT in ob/ob mice treated with NS or DOP (E-F). The GTTs and ITTs in ob/ob mice treated with NS or DOP ( $n=5$ per group). AUC: area under curve. Data are presented as mean \pm SD. Statistical significance was calculated using the two-tailed Student's t-test. * means $p<0.05$.

\section{ETHICS STATEMENT}

The animal study was reviewed and approved by the Institutional Animal Care and Use Committee of the Laboratory Animal Research Center at Xiangya Medical School of Central South University, China.

\section{AUTHOR CONTRIBUTIONS}

Study design: QL, SH; Study conduct: XX, WW, HZ, HL, and JW; Data collection: XX, WW, ZZ, HL, HZ, and JW; Data analysis: $\mathrm{QL}, \mathrm{XX}, \mathrm{XL}$, and JQ; Data interpretation: QL, XX, XL, and JQ; Drafting manuscript: XX and WW; Revising manuscript content:

\section{REFERENCES}

Barazzoni, R., Gortan Cappellari, G., Ragni, M., and Nisoli, E. (2018). Insulin Resistance in Obesity: an Overview of Fundamental Alterations. Eat. Weight Disord. 23 (2), 149-157. doi:10.1007/s40519-018-0481-6

Beale, E. G. (2013). Insulin Signaling and Insulin Resistance. J. Investig. Med. 61 (1), 11-14. doi:10.2310/JIM.0b013e3182746f95

Belkina, A. C., and Denis, G. V. (2010). Obesity Genes and Insulin Resistance. Curr. Opin. Endocrinol. Diabetes Obes. 17 (5), 472-477. doi:10.1097/MED. 0b013e32833c5c48

Boden, G., Chen, X., Ruiz, J., White, J. V., and Rossetti, L. (1994). Mechanisms of Fatty Acid-Induced Inhibition of Glucose Uptake. J. Clin. Invest. 93 (6), 2438-2446. doi:10.1172/JCI117252

Council of the Obesity, S. (2008). Obesity as a Disease: the Obesity Society Council Resolution. Obesity (Silver Spring) 16 (6), 1151. doi:10.1038/oby.2008.246
QL, XX, ST; Approval of the final manuscript: QL, XX, WW, $\mathrm{HZ}, \mathrm{HL}, \mathrm{XL}, \mathrm{JW}, \mathrm{ZZ}$, and $\mathrm{SH}$ are responsible for the integrity of the data analysis, and all authors take responsibility for and attest to the integrity of the data analysis. QL and SH are responsible for sharing the editors' comments with the other authors.

\section{FUNDING}

This work was supported by the Natural Science Foundation of Hunan Province (2020JJ4799) and National Science and Technology Major Project (Grant No:2020ZX09201-28).

Crowe, S., Wu, L. E., Economou, C., Turpin, S. M., Matzaris, M., Hoehn, K. L., et al. (2009). Pigment Epithelium-Derived Factor Contributes to Insulin Resistance in Obesity. Cel Metab. 10 (1), 40-47. doi:10.1016/j.cmet.2009. 06.001

Czech, M. P. (2017). Insulin Action and Resistance in Obesity and Type 2 Diabetes. Nat. Med. 23 (7), 804-814. doi:10.1038/nm.4350

De Lorenzo, A., Gratteri, S., Gualtieri, P., Cammarano, A., Bertucci, P., and Di Renzo, L. (2019). Why Primary Obesity Is a Disease?. J. Transl Med. 17 (1), 169 doi:10.1186/s12967-019-1919-y

Dubuc, P. U. (1976). The Development of Obesity, Hyperinsulinemia, and Hyperglycemia in Ob/ob Mice. Metabolism. 25 (12), 1567-1574. doi:10. 1016/0026-0495(76)90109-8

Esposito, K., Pontillo, A., Giugliano, F., Giugliano, G., Marfella, R., Nicoletti, G., et al. (2003). Association of Low Interleukin-10 Levels with the Metabolic Syndrome in Obese Women. J. Clin. Endocrinol. Metab. 88 (3), 1055-1058. doi:10.1210/jc.2002-021437 
Fellmann, L., Nascimento, A. R., Tibiriça, E., and Bousquet, P. (2013). Murine Models for Pharmacological Studies of the Metabolic Syndrome. Pharmacol. Ther. 137 (3), 331-340. doi:10.1016/j.pharmthera.2012.11.004

Frayn, K. N. (1993). Insulin Resistance and Lipid Metabolism. Curr. Opin. Lipidol. 4 (3), 197-204. doi:10.1097/00041433-199306000-00004

Germoush, M. O., Elgebaly, H. A., Hassan, S., Kamel, E. M., Bin-Jumah, M., and Mahmoud, A. M. (2019). Consumption of Terpenoids-Rich Padina Pavonia Extract Attenuates Hyperglycemia, Insulin Resistance and Oxidative Stress, and Upregulates PPAR $\gamma$ in a Rat Model of Type 2 Diabetes. Antioxidants. 9 (1), 22. doi:10.3390/antiox9010022

Ghorpade, D. S., Ozcan, L., Zheng, Z., Nicoloro, S. M., Shen, Y., Chen, E., et al. (2018). Hepatocyte-secreted DPP4 in Obesity Promotes Adipose Inflammation and Insulin Resistance. Nature. 555 (7698), 673-677. doi:10.1038/nature26138

González-Domínguez, Á., Visiedo-García, F. M., Domínguez-Riscart, J., GonzálezDomínguez, R., Mateos, R. M., and Lechuga-Sancho, A. M. (2020). Iron Metabolism in Obesity and Metabolic Syndrome. Int. J. Mol Sci. 21 (15), 5529. doi:10.3390/ijms21155529

Gortan Cappellari, G., Zanetti, M., Semolic, A., Vinci, P., Ruozi, G., Falcione, A., et al. (2016). Unacylated Ghrelin Reduces Skeletal Muscle Reactive Oxygen Species Generation and Inflammation and Prevents High-Fat Diet-Induced Hyperglycemia and Whole-Body Insulin Resistance in Rodents. Diabetes. 65 (4), 874-886. doi:10.2337/db15-1019

Guerre-Millo, M., Rouault, C., Poulain, P., Andre, J., Poitout, V., Peters, J. M., et al. (2001). PPAR- -Null Mice Are Protected from High-Fat Diet-Induced Insulin Resistance. Diabetes. 50 (12), 2809-2814. doi:10.2337/diabetes.50. 12.2809

Hotamisligil, G., Shargill, N., and Spiegelman, B. (1993). Adipose Expression of Tumor Necrosis Factor-Alpha: Direct Role in Obesity-Linked Insulin Resistance. Science. 259 (5091), 87-91. doi:10.1126/science.7678183

Hua, Y.-F., Zhang, M., Fu, C.-X., Chen, Z.-H., and Chan, G. Y. S. (2004). Structural Characterization of a 2-O-Acetylglucomannan from Dendrobium Officinale Stem. Carbohydr. Res. 339 (13), 2219-2224. doi:10.1016/j.carres.2004.05.034

Huang, K., Li, Y., Tao, S., Wei, G., Huang, Y., Chen, D., et al. (2016). Purification, Characterization and Biological Activity of Polysaccharides from Dendrobium Officinale. Molecules. 21 (6), 701. doi:10.3390/molecules21060701

Jastreboff, A. M., Kotz, C. M., Kahan, S., Kelly, A. S., and Heymsfield, S. B. (2019). Obesity as a Disease: The Obesity Society 2018 Position Statement. Obesity. 27 (1), 7-9. doi:10.1002/oby.22378

Kern, P. A., Ranganathan, S., Li, C., Wood, L., and Ranganathan, G. (2001). Adipose Tissue Tumor Necrosis Factor and Interleukin-6 Expression in Human Obesity and Insulin Resistance. Am. J. Physiology-Endocrinology Metab. 280 (5), E745-E751. doi:10.1152/ajpendo.2001.280.5.E745

Klop, B., Elte, J., and Cabezas, M. (2013). Dyslipidemia in Obesity: Mechanisms and Potential Targets. Nutrients. 5 (4), 1218-1240. doi:10.3390/nu5041218

Kojta, I., Chacińska, M., and Błachnio-Zabielska, A. (2020). Obesity, Bioactive Lipids, and Adipose Tissue Inflammation in Insulin Resistance. Nutrients. 12 (5), 1305. doi:10.3390/nu12051305

Laaksonen, D. E., Lakka, H. M., Niskanen, L. K., Kaplan, G. A., Salonen, J. T., and Lakka, T. A. (2002). Metabolic Syndrome and Development of Diabetes Mellitus: Application and Validation of Recently Suggested Definitions of the Metabolic Syndrome in a Prospective Cohort Study. Am. J. Epidemiol. 156 (11), 1070-1077. doi:10.1093/aje/kwf145

Lee, Y. S., Kim, J.-W., Osborne, O., Oh, D. Y., Sasik, R., Schenk, S., et al. (2014). Increased Adipocyte O2 Consumption Triggers HIF-1 $\alpha$, Causing Inflammation and Insulin Resistance in Obesity. Cell. 157 (6), 1339-1352. doi:10.1016/j.cell. 2014.05.012

Li, H., Xie, H., Liu, W., Hu, R., Huang, B., Tan, Y.-F., et al. (2009). A Novel microRNA Targeting HDAC5 Regulates Osteoblast Differentiation in Mice and Contributes to Primary Osteoporosis in Humans. J. Clin. Invest. 119 (12), 3666-3677. doi:10.1172/JCI39832

Liang, J., Chen, S., Chen, J., Lin, J., Xiong, Q., Yang, Y., et al. (2018). Therapeutic Roles of Polysaccharides from Dendrobium Officinaleon Colitis and its Underlying Mechanisms. Carbohydr. Polym. 185, 159-168. doi:10.1016/j. carbpol.2018.01.013

Liang, J., Li, H., Chen, J., He, L., Du, X., Zhou, L., et al. (2019). Dendrobium Officinale Polysaccharides Alleviate colon Tumorigenesis via Restoring Intestinal Barrier Function and Enhancing Anti-tumor Immune Response. Pharmacol. Res. 148, 104417. doi:10.1016/j.phrs.2019.104417
Ling, C., and Rönn, T. (2019). Epigenetics in Human Obesity and Type 2 Diabetes. Cel Metab. 29 (5), 1028-1044. doi:10.1016/j.cmet.2019.03.009

Lu, M., Sarruf, D. A., Talukdar, S., Sharma, S., Li, P., Bandyopadhyay, G., et al. (2011). Brain PPAR- $\gamma$ Promotes Obesity and Is Required for the InsulinSensitizing Effect of Thiazolidinediones. Nat. Med. 17 (5), 618-622. doi:10. $1038 / \mathrm{nm} .2332$

Lumeng, C. N., Bodzin, J. L., and Saltiel, A. R. (2007). Obesity Induces a Phenotypic Switch in Adipose Tissue Macrophage Polarization. J. Clin. Invest. 117 (1), 175-184. doi:10.1172/JCI29881

Meigs, J. B., Wilson, P. W. F., Fox, C. S., Vasan, R. S., Nathan, D. M., Sullivan, L. M., et al. (2006). Body Mass index, Metabolic Syndrome, and Risk of Type 2 Diabetes or Cardiovascular Disease. J. Clin. Endocrinol. Metab. 91 (8), 2906-2912. doi:10.1210/jc.2006-0594

Moller, D. E., and Kaufman, K. D. (2005). Metabolic Syndrome: a Clinical and Molecular Perspective. Annu. Rev. Med. 56, 45-62. doi:10.1146/annurev.med. 56.082103 .104751

Ormazabal, V., Nair, S., Elfeky, O., Aguayo, C., Salomon, C., and Zuñiga, F. A. (2018). Association between Insulin Resistance and the Development of Cardiovascular Disease. Cardiovasc. Diabetol. 17 (1), 122. doi:10.1186/ s12933-018-0762-4

Randle, P. J., Garland, P. B., Hales, C. N., and Newsholme, E. A. (1963). The Glucose Fatty-Acid Cycle its Role in Insulin Sensitivity and the Metabolic Disturbances of Diabetes Mellitus. The Lancet. 281 (7285), 785-789. doi:10. 1016/s0140-6736(63)91500-9

Randle, P. J., Garland, P. B., Newsholmet, E. A., and Hales, C. N. (1965). The Glucose Fatty Acid Cycle in Obesity and Maturity Onset Diabetes Mellitus. Ann. NY Acad. Sci. 131 (1), 324-333. doi:10.1111/j.1749-6632.1965.tb34800.x

Randle, P., Newsholme, E., and Garland, P. (1964). Regulation of Glucose Uptake by Muscle. 8. Effects of Fatty Acids, Ketone Bodies and Pyruvate, and of Alloxan-Diabetes and Starvation, on the Uptake and Metabolic Fate of Glucose in Rat Heart and Diaphragm Muscles. Biochem. J. 93 (3), 652-665. doi:10.1042/ bj0930652

Reaven, G. M., Hollenbeck, C., Jeng, C. Y., Wu, M. S., and Chen, Y. D. (1988). Measurement of Plasma Glucose, Free Fatty Acid, Lactate, and Insulin for $24 \mathrm{H}$ in Patients with NIDDM. Diabetes. 37 (8), 1020-1024. doi:10.2337/diab.37.8. 102010.2337/diabetes.37.8.1020

Samuel, V. T., and Shulman, G. I. (2012). Mechanisms for Insulin Resistance: Common Threads and Missing Links. Cell. 148 (5), 852-871. doi:10.1016/j.cell. 2012.02.017

Savage, D. B., Petersen, K. F., and Shulman, G. I. (2007). Disordered Lipid Metabolism and the Pathogenesis of Insulin Resistance. Physiol. Rev. 87 (2), 507-520. doi:10.1152/physrev.00024.2006

Semple, R. K., Chatterjee, V. K., and O'Rahilly, S. (2006). PPAR and Human Metabolic Disease. J. Clin. Invest. 116 (3), 581-589. doi:10.1172/JCI28003

Su, T., Xiao, Y., Xiao, Y., Guo, Q., Li, C., Huang, Y., et al. (2019). Bone Marrow Mesenchymal Stem Cells-Derived Exosomal MiR-29b-3p Regulates AgingAssociated Insulin Resistance. ACS Nano. 13 (2), 2450-2462. doi:10.1021/ acsnano.8b09375

Taylor, E. B. (2021). The Complex Role of Adipokines in Obesity, Inflammation, and Autoimmunity. Clin. Sci. (Lond). 135 (6), 731-752. doi:10.1042/ CS20200895

Teixeira da Silva, J. A., Tsavkelova, E. A., Zeng, S., Ng, T. B., Parthibhan, S., Dobránszki, J., et al. (2015). Symbiotic In Vitro Seed Propagation of Dendrobium: Fungal and Bacterial Partners and Their Influence on Plant Growth and Development. Planta. 242 (1), 1-22. doi:10.1007/s00425-0152301-9

Tomita, T., Doull, V., Pollock, H. G., and Krizsan, D. (1992). Pancreatic Islets of Obese Hyperglycemic Mice (Ob/ob). Pancreas. 7 (3), 367-375. doi:10.1097/ 00006676-199205000-00015

Winer, D. A., Luck, H., Tsai, S., and Winer, S. (2016). The Intestinal Immune System in Obesity and Insulin Resistance. Cel Metab. 23 (3), 413-426. doi:10. 1016/j.cmet.2016.01.003

Wu, H., and Ballantyne, C. M. (2017). Skeletal Muscle Inflammation and Insulin Resistance in Obesity. J. Clin. Invest. 127 (1), 43-54. doi:10.1172/JCI88880

Xiao, Y.-Z., Yang, M., Xiao, Y., Guo, Q., Huang, Y., Li, C.-J., et al. (2020). Reducing Hypothalamic Stem Cell Senescence Protects against Aging-Associated Physiological Decline. Cel Metab. 31 (3), 534-548 e535. doi:10.1016/j.cmet. 2020.01.002 
Xu, H., Barnes, G. T., Yang, Q., Tan, G., Yang, D., Chou, C. J., et al. (2003). Chronic Inflammation in Fat Plays a Crucial Role in the Development of ObesityRelated Insulin Resistance. J. Clin. Invest. 112 (12), 1821-1830. doi:10.1172/ JCI1945110.1172/jci200319451

Yang, J., Chen, H., Nie, Q., Huang, X., and Nie, S. (2020). Dendrobium Officinale Polysaccharide Ameliorates the Liver Metabolism Disorders of Type II Diabetic Rats. Int. J. Biol. Macromolecules. 164, 1939-1948. doi:10.1016/j.ijbiomac.2020. 08.007

Yee, H. Y., Yang, J. J., Wan, Y. G., Chong, F. L., Wu, W., Long, Y., et al. (2019). Molecular Mechanisms of Insulin Resistance and Interventional Effects of Chinese Herbal Medicine. Zhongguo Zhong Yao Za Zhi. 44 (7), 1289-1294. doi:10.19540/j.cnki.cjcmm.20181105.003

Ying, W., Riopel, M., Bandyopadhyay, G., Dong, Y., Birmingham, A., Seo, J. B., et al. (2017). Adipose Tissue Macrophage-Derived Exosomal miRNAs Can
Modulate In Vivo and In Vitro Insulin Sensitivity. Cell. 171 (2), 372-384 e312. doi:10.1016/j.cell.2017.08.035

Conflict of Interest: The authors declare that the research was conducted in the absence of any commercial or financial relationships that could be construed as a potential conflict of interest.

Copyright $\odot 2021 \mathrm{Qu}, \mathrm{Tan}, \mathrm{Xie}, \mathrm{Wu}, \mathrm{Zhu}, \mathrm{Li}$, Liao, Wang, Zhou, Huang and Lu. This is an open-access article distributed under the terms of the Creative Commons Attribution License (CC BY). The use, distribution or reproduction in other forums is permitted, provided the original author(s) and the copyright owner(s) are credited and that the original publication in this journal is cited, in accordance with accepted academic practice. No use, distribution or reproduction is permitted which does not comply with these terms. 